\title{
REAL-TIME TISSUE ELASTOGRAPHY FOR THE DIAGNOSIS OF LYMPH NODE METASTASIS IN ORAL SQUAMOUS CELL
}

\section{CARCINOMA}

NaOmi Ishibashi, Kenji Yamagata, Hiroyoshi Sasaki, Kahori Seto, YoshiKo Shinya,

Hiroyuki Ito, Keju ShinozuKa, Toru Yanagawa, KoJiro OnizaWa, Hiroki Bukawa

Oral and Maxillofacial Surgery, Clinical Sciences, Graduate School of Comprehensive

Human Sciences, University of Tsukuba

Address correspondence to Naomi Ishibashi, Oral and Maxillofacial Surgery, Clinical Sciences, Graduate School of Comprehensive Human Sciences, University of Tsukuba, 1-1-1 Tennodai, Tsukuba, Ibaraki, 305-8575, Japan. E-mail: greened_amethyst829@hotmail.com Tel: +81-29-853-3052, Fax: +81-29-853-3052 


\begin{abstract}
We compared conventional ultrasound (US) B-mode, color Doppler, and elastographic assessment of lymph node (LN) stiffness against pathological findings from surgical samples, to determine the most useful factors for identifying LN metastases. Seventy-one LNs in 19 patients with oral squamous cell carcinoma (OSCC) were examined. Using our new system, elastography images were scored from 1-5. The score 1-4 were correlate with the blue area of each LN, which indicated increased stiffness: (1) none; (2) <50\%; (3) 50\%; or (4) >50\%. A score 5 indicated central necrosis, and did not correlate with the blue area. We found significant differences in minimal diameter, shape index, margin, internal structure, hilus presence or absence, elastography score, and percentage of blue area between metastatic and non-metastatic LNs. Stepwise regression analysis identified elastography score 3-5 as an independent significant LN metastatic factor, suggesting that our scoring system may be useful for accurately diagnosing metastatic LNs.
\end{abstract}

Key Words: Ultrasonography, Elastography, Oral cancer, LN metastasis, Diagnosis 


\section{INTRODUCTION}

Diagnosing lymph node (LN) metastasis is important in determining prognosis and selecting treatment strategies for patients with oral cancer. Metastasis indications related to LN size, stiffness, shape, internal properties, and adhesion to surrounding tissue have generally been evaluated by palpation, computed tomography (CT), magnetic resonance imaging (MRI), and ultrasonography (US). Palpation, which has been a primary method of judging LN stiffness, is subjective, depending largely on the experience and objectivity of each examiner, and is especially difficult in the case of small or deep LNs.

Real-time tissue elastography, although a comparatively new technique, is now available on conventional US systems. Tissue stiffness is estimated by comparing local tissue displacement from ultrasonic signals with and without tissue compression; soft tissues show deformity or strain more readily than stiff tissues when compressed. US elastography has been used to evaluate lesions surrounded by soft tissue in the breast, thyroid, prostate, liver, cervix and pancreas. Elastography is well-suited to examining cervical LNs, which are easily accessible and can be compressed against underlying anatomic structures with the US probe. LN stiffness measurements are clinically useful for accurately diagnosing metastatic LNs. To our knowledge, only four articles 
concerning the use of elastography in diagnosing cervical LN metastasis have been published ( Alam et al. 2008; Bhatia et al. 2010; Lyshchik et al. 2007; Tan et al. 2010).

The objective of this study was to evaluate the association between elastographic LN stiffness measurements and the metastatic state of LNs, and to determine the usefulness of our new elastography score in diagnosing metastatic LNs.

\section{MATERIALS AND METHODS}

Patients

This study examined 71 LNs in 19 consecutive patients with a clinical and histopathological diagnosis of oral squamous cell carcinoma (OSCC). The study included 13 male and 6 female patients ranging in age from 57 to 88 years, with an average of 69.9 years. These patients underwent neck dissection without preoperative treatment at the Department of Oral and Maxillofacial Surgery, Tsukuba University Hospital, between November 2008 and August 2010. The informed consent was obtained from each patient and this study approved by our university hospital's ethics committee. Primary tumor sites were found in the tongue (10), the lower gingiva (7), the buccal mucosa (1), and the mouth floor (1). UICC clinical tumor stages were assessed as follows: Stage I (1), Stage II (8), Stage III (6), and Stage IV (4). Histopathologically, 
five patients had only metastatic LNs, 5 had only non-metastatic LNs and 9 had both LNs.

US examinations

US B-mode, color Doppler, and elastography scans were performed transcutaneously on all patients before neck dissection, and the results were compared to the postoperative histopathological diagnosis. US LN images were recorded both on film and in DICOM files. B-mode imaging was used to ascertain LN maximal and minimal diameters, shape index (minimal/maximal diameter), margin patterns, internal echoes, and the presence or absence of a hilus. Color Doppler imaging was used to determine the presence of blood flow to the hilus; and elastography imaging was used to evaluate LN stiffness. Two oral and maxillofacial surgeons (N.I. and K.Y.), each with at least 2 years of practical experience performing elastography in the neck region without variability between two operators, performed the scans using an ultrasound scanner (EUB-8500, Hitachi Medical Corporation, Tokyo, Japan) and a 14-6 MHz linear probe (Hitachi EUP-L65). When the discrepancy occurred between two operators, the decision was made after due consultation.

Although, in general, the minimal diameters of metastatic LNs were assumed to 
be less than $10 \mathrm{~mm}$ by a clinical scene of the head and neck cancer, they are often overlooked due to the impossibility of detection by palpation, CT, or MRI, and the careful diagnosis of LNs becomes possible for the level of $10 \mathrm{~mm}$ or less and $6 \mathrm{~mm}$ or more is defined as suspected of being positive for metastasis by US (Furukawa and Furukawa 2010). In this study, the border of minimal diameter decided in the metastatic group of 8mm or more, non-metastatic group of less than 8mm (Rin et al. 2010).

Elastography was performed with the patient lying in the same position as for conventional US examination. Conventional B-mode and color Doppler images of target LNs were obtained first, followed by the elastography images. The region of interest (ROI) for elastography was set to include sufficient surrounding fat tissue for elasticity to be displayed relative to the average strain inside the ROI. The probe was applied perpendicular to the neck and used to compress the tissue repeatedly. Each pixel of the elasticity image was assigned one of 256 specific colors depending on the magnitude of strain, with red representing the softest components and blue representing the hardest. Average strain in the ROI was indicated in green. Compression was limited to the range resulting in red to green being assigned to the surrounding fat tissue in the ROI. No patients in this study experienced adverse events from either conventional US or elastography scans. 


\section{Evaluation}

We assigned elastography images to five categories according to a modification of a previously reported scoring system (Alam et al. 2008), as follows: Score 1. no blue area; Score 2. small blue area; Score 3. half of LN colored blue; Score 4. more than half of the LN colored blue; Score 5. a peripheral blue area and a central red, yellow, or green area (indicating obvious central necrosis (Table 1). Clinically, the elastography score was judged at the time of US examination in externals and noted in the ultrasound reports.

To evaluate the accuracy of our scoring system, one observer (T.Y) measured the percentage of blue area in the target LNs for later statistical and objective evaluation against the elastography score and the pathological diagnosis as follows; At the Elastography mode, an elastography image is displayed on the left side of screen and a B-mode image on the right side. To obtain the data, first, the outline of the target LN was drawn and determined on the B-mode image, and then that outline put on the elastography image. Color of this area were decomposed into R(red), G(green), and B(blue), and measured the percentage of blue area automatically with RGB histogram by Image $\mathrm{J}$ image analysis software, developed by the National Institutes of Health, and 
found under image processing and analysis at Java.rsb.info.nih.gov/ij/.

All patients underwent neck dissection and all visible and palpable lymph nodes were carefully examined in neck dissection specimens by authors to identify whether or not they were detected sonographically. Although all nodes were removed and subjected to histologic examination, only the nodes that correlated with the ultrasonograms served as materials for this study. Malignant LN involvement was diagnosed by experienced pathologists, and the US findings were compared to the histopathological diagnosis. Diagnostic sensitivity, specificity, accuracy, positive predictive value (PPV), and negative predictive value (NPV) were calculated.

\section{Statistical analysis}

Calculations and statistical analyses were performed using the Stat View 5.0 J (SAS Institute Inc., U.S.A.) software package. The Mann-Whitney U-test and Chi-square test or Fisher's exact probability test were used in statistical comparisons between metastatic and non-metastatic LNs. Spearman's correlation coefficient by rank test was used in the statistical correlation between the elastography score and the percentage of blue area in the LN. Stepwise regression analysis was applied to variables assessed as significant with univariate analysis. Logistic regression analysis was 
performed to determine odds ratios (OR) with 95\% confidence intervals. The significance level was recognized as $\mathrm{P}<0.05$ for all values.

\section{RESULTS}

\section{Conventional ultrasonography}

Of the 71 cervical LNs evaluated by US prior to neck dissection, 31 (43.7\%) were diagnosed as metastatic, and 40 (56.3\%) as non-metastatic according to ultrasonographic LN metastasis criteria, based on the minimal diameter, shape, and margin, and changes in the internal structure and blood flow.

The minimal LN diameter ranged from 3.2 to $19.2 \mathrm{~mm}$ in the metastatic group, with a median of $9.1 \mathrm{~mm}$, and from 2.4 to $8.3 \mathrm{~mm}$ in the non-metastatic group, with a median of $4.0 \mathrm{~mm}$. Minimal diameters were significantly larger for metastatic than non-metastatic LNs $(\mathrm{P}<0.01)$, with 16 LNs in the metastatic group having a minimal diameter of $8 \mathrm{~mm}$ or more. There was a significant difference in histological diagnosis between LNs with a minimal diameter $8 \mathrm{~mm}$ or more and those $<8 \mathrm{~mm}(\mathrm{P}<0.01)$. The shape index (minimal/maximal diameter) ranged from 0.27 to 0.86 for the metastatic group, with a median of 0.55 , and from 0.18 to 0.79 for the non-metastatic group, with a median of 0.37. Metastatic LNs were significantly more spherical in shape than 
non-metastatic LNs $(\mathrm{P}<0.01)$. All LNs with irregular margins or heterogeneous internal echo patterns were found to be metastatic. There was no hilus present in 25 of the metastatic and 19 of the non-metastatic LNs. There were significant differences in LN margin, internal echo, and the absence of hilus. In the color Doppler images, blood flow to the hilus was absent in 15 metastatic and 7 non-metastatic LNs, with no significant difference (Table 2).

\section{Elastography}

The median elastography score was 4 for the metastatic LN group, and 2 for the non-metastatic LN group. Table 3 shows the frequency and percentage of elastography scores grouped by the histological determination; there was a significant difference in LN elastography scores between metastatic and non-metastatic LN groups $(\mathrm{P}<0.01)$. The most frequent score in the metastatic group was 4 (17 LNs; 54.9\%). On the other hand, the most frequent score in the non-metastatic group was 2 (25 LNs; 62.5\%). The frequency of a score of 3 was almost the same in the two groups. Patients with scores of 1 and 2 were classed as elastographic-negative, and those with scores of 3-5 were classed as elastographic-positive. Twenty-six metastatic LNs were observed in the elastographic-positive group, and 5 in the elastographic-negative group. There was a 
significant difference in histological diagnosis between the elastographic-positive and -negative LN groups $(\mathrm{P}<0.01)$ (Table 4).

The median percentage of blue area in the target LN, measured by Image $\mathrm{J}$ image analysis software, was $61.6 \%$ for the metastatic group and $36.7 \%$ for the non-metastatic group. Box plots of the percentage of blue area in the LNs divided according to histological diagnosis are shown in Figure 1. We decided the 50\% threshold according to the criterion of elastography score 3 or 4 with half of $\mathrm{LN}$ colored blue. There was a significant difference in histological diagnosis when groups were divided at the threshold of $50 \%$ blue area in the $\mathrm{LN}(\mathrm{P}<0.01)$ (Table 5). When grouped by elastography score, LNs with scores of 1-4 showed a positive correlation with the statistical percentage value of the blue area in the LN (Spearman coefficient 0.82; $\mathrm{P}<0.001)$. The percentage value of the blue area in the group with a score of 5 reflected the degree of central necrosis in the LN.

To find the best parameters for predicting LN metastasis, we evaluated the diagnostic precision of various factors separately and combined. First, based on previously reported information, we evaluated the presence of 2 factors, a minimal diameter of $8 \mathrm{~mm}$ or more, or a heterogeneous iso- or hyper-echoic signal in the B-mode image, as a predictor of metastasis (Furukawa and Furukawa 2010; Rin et al. 
2010). The two-factor prediction gave a sensitivity of $70.97 \%$, specificity of $97.50 \%$, and accuracy of $85.92 \%$. The PPV (positive predictive value) was 95.65\%, and the NPV (negative predictive value) was $81.25 \%$. If an elastography score of 3 or more was assumed to indicate metastasis, the precision of diagnosis was as follows: sensitivity, 83.87\%; specificity, 82.50\%; accuracy, 83.10\%; PPV, 78.79\%; and NPV, 86.80\%. If a minimal diameter of $8 \mathrm{~mm}$ or greater, heterogeneous iso- or hyper-echoic signal in B-mode image, or an elastography score of 3-5 were used in combination to indicate metastasis, the precision of diagnosis was as follows: sensitivity, $90.32 \%$; specificity, 80\%; accuracy, 84.51\%; PPV, 77.78\%; and NPV, 91.43\% (Table 6).

Factors associated with multivariate analysis

Stepwise regression analysis showed that an elastography score of 3-5 was an independent, significant factor among the seven variables assessed as significant with univariate analysis: minimal diameter, shape index, pattern of internal echo, echogenic hilus, margin, elastography score, and percentage of blue area in the LN. Logistic regression analysis of this independent variable showed that the hazard ratio (95\% confidence interval) was 18.2 (2.79 - 119.2) for elastography scores of 3-5 in positive LNs (Table 7). 


\section{DISCUSSION}

Diagnosing cervical LN metastasis in OSCC patients is important in determining the extent of necessary neck dissection as well as the prognosis. However, diagnosing metastatic LNs with a minor diameter of less than $10 \mathrm{~mm}$ is difficult, as they cannot be evaluated by CT, MRI, or indeed any method other than US. Although US cannot be used to evaluate micrometastases, it still offers a higher degree of accuracy than other available methods (Furukawa and Furukawa 2010). There are two scenarios that occur in cases of LN metastasis - either the metastatic foci are completely confined to the LNs, or there is extra-nodal spread. Cases of clinical extra-nodal metastatic spread are comparatively easy to diagnose by CT, MRI, US, or other methods. However, it is very difficult, or even impossible, to ascertain the presence of micrometastatic foci inside LNs, even by US.

Metastases develop within LNs before the nodes enlarge. Metastatic cancer cells invade an LN through afferent lymphatic vessels, and a metastatic focus initially forms in a peripheral region remote from the hilus of the LN. The displaced fatty hilus becomes localized after the metastatic focus becomes larger. The first changes in the LN that can be detected by US are an increased thickness of the node, including a thickened 
LN capsule (Furukawa and Furukawa 2010). However, it is still difficult to obtain convincing evidence of LN metastasis at this stage, using US B-mode, color Doppler, or elastography.

Conventional US examinations consisting of B-mode and color Doppler images have been widely used for many years to detect metastatic LNs in cases of OSCC. In recent years, elastography has become useful for evaluating tissue stiffness compared with surrounding tissue in a region of interest (ROI). Elastography has been studied in the breast (Itoh et al. 2006; Zhu et al. 2008), thyroid gland (Lyshchik et al. 2005), prostate (Miyagawa et al. 2009), liver (Gomez-Dominguez et al. 2006), pancreas (Giovannini et al. 2009), and cervix (Thomas et al. 2007). When target lesions are surrounded with soft tissue, elastography evaluation is an appropriate examination tool. Anatomically, cervical LNs are well-positioned for elastographic examination. A report comparing reactive LNs with metastatic LNs (Alam et al. 2008) found that discriminating reactive LNs was difficult when using only information obtained with B-mode imaging - minimal diameter, shape, pattern of internal echo, existence of hilus, etc. Combined evaluation with elastography and B-mode images was found to be the most effective.

There have been a few reports on classifying elastography images in cervical LN 
lesions (Alam et al. 2008; Taira et al. 2008). Although one classification system is based on the percentage of blue area in the target LN, estimated visually, visual assessment alone is subjective and unlikely to be comparable from study to study (or physician to physician). To remedy this deficit, we introduced an objective measurement, using Image $\mathrm{J}$ image analysis software. Although an elastography score of 1-4 was positively correlated with the percentage of the ROI that was blue, this correlation was lost for LNs with an elastography score of 5, which indicates central necrosis. LNs with central necrosis were clinically diagnosed as metastatic by CT and other methods, and the presence of cancer cells and necrotic tissue was confirmed histologically by experienced pathologists. A large necrotic area in an LN might be detected as a soft central area (red, yellow or/and green), and therefore the percentage of blue area was not an accurate predictor of metastasis for these LNs.

Table 4 compares LNs divided into two groups according to the elastography score (1-2, and 3-5). The frequency of a score of 3 was almost same between metastatic and non-metastatic LNs. Since false positives are less consequential for the patient than false negatives, we defined a score of 1 or 2 as elastographic-negative, and a score of 3 to 5 as elastographic-positive.

As noted above, the elastographic ROI must be set to include sufficient 
surrounding fat tissue, because elasticity is displayed relative to the average strain inside the ROI. The compression strength is limited to that resulting in a green to red color being assigned to the fat tissue surrounding the target in the ROI. The strain ratio, or ratio of the average strain of the target area to that of the surrounding tissue, can be calculated (Lyshchik et al. 2007; Rin et al. 2010; Thomas et al. 2010; Zhi et al. 2010). Zhi et al.(2010) reported that the strain ratio assessment could be a more reliable predictor than the five-point scoring method (Zhu et al. 2008) for determining breast malignancy with elastography. On the other hand, limitations of using the strain ratio in evaluating breast lesions have also been reported (Lyshchik et al. 2007), including the patient's movement being a potential source of error. The presence of central necrosis may also impair the differentiation of lesions by a subjective interpretation of the elastography score according to the strain ratio. In the future, it may be possible to find an objective basis for comparison between the strain of the target area and that of the surrounding tissue, but currently the use of the strain ratio requires further consideration when it comes to evaluating cervical lesions.

In the present study, we were able to obtain better sensitivity and NPV using both B-mode and elastography than with either method alone. In the multivariate analysis, an elastography score of 3 to 5 was selected as an independent significant factor. When 
target lesions are surrounded with soft tissue, elastography can be used as an appropriate examination tool. Furthermore, the LN elastography scores provided useful information beyond that provided by B-mode imaging alone.

Elastography is an effective supplement to conventional ultrasonography. The results of our study indicated that our elastography classification score was a useful diagnostic factor, especially in combination with other factors. LN evaluation with both elastography and B-mode imaging is clinically recommended for more accurate diagnosis of metastatic LNs. 


\section{REFERENCES}

Alam F, Naito K, Horiguchi J, Fukuda H, Tachikake T, Ito K. Accuracy of sonographic elastography in the differential diagnosis of enlarged cervical lymph nodes: comparison with conventional B-mode sonography. AJR Am J Roentgenol 2008;191:604-10.

Bhatia KS, Cho CC, Yuen YH, Rasalkar DD, King AD, Ahuja AT. Real-time qualitative ultrasound elastography of cervical lymph nodes in routine clinical practice: interobserver agreement and correlation with malignancy. Ultrasound Med Biol 2010;36:1990-7.

Furukawa MK, Furukawa M. Diagnosis of lymph node metastases of head and neck cancer and evaluation of effects of chemoradiotherapy using ultrasonography. Int J Clin Oncol 2010;15:23-32.

Giovannini M, Thomas B, Erwan B, Christian P, Fabrice C, Benjamin E, Genevieve M, Paolo A, Pierre D, Robert Y, Walter S, Hanz S, Carl S, Christoph D, Pierre E, Jean-Luc VL, Jacques D, Peter V, Andrian S. Endoscopic ultrasound elastography for evaluation of lymph nodes and pancreatic masses: a multicenter study. World J Gastroenterol 2009;15:1587-93.

Gomez-Dominguez E, Mendoza J, Rubio S, Moreno-Monteagudo JA, Garcia-Buey L, Moreno-Otero R. Transient elastography: a valid alternative to biopsy in patients with chronic liver disease. Aliment Pharmacol Ther 2006;24:513-8.

Itoh A, Ueno E, Tohno E, Kamma H, Takahashi H, Shiina T, Yamakawa M, Matsumura T. Breast disease: clinical application of US elastography for diagnosis. Radiology 2006;239:341-50.

Lyshchik A, Higashi T, Asato R, Tanaka S, Ito J, Hiraoka M, Insana MF, Brill AB, Saga T, Togashi K. Cervical lymph node metastases: diagnosis at sonoelastography--initial experience. Radiology 2007;243:258-67.

Lyshchik A, Higashi T, Asato R, Tanaka S, Ito J, Mai JJ, Pellot-Barakat C, Insana MF, Brill AB, Saga T, Hiraoka M, Togashi K. Thyroid gland tumor diagnosis at US elastography. Radiology 2005;237:202-11.

Miyagawa T, Tsutsumi M, Matsumura T, Kawazoe N, Ishikawa S, Shimokama T, Miyanaga N, Akaza H. Real-time elastography for the diagnosis of prostate cancer: evaluation of elastographic moving images. Jpn J Clin Oncol 2009;39:394-8. 
Rin S, Yamashita T, Ueda M, asaka Y, Nakajima Y, Tochihara Y, Ishiyama T, Hosokawa S, Yahara M, Kubo Y, Murakami M, Hujita M, Kitamura T. Usefulness of investigation with real-time tissue elastography to diagnose cervical lymph node metastasis in head and neck cancer. Head and Neck Cancer 2010;36:349.

Taira S, Nikkuni Y, Hayashi T, Hoshina H, Shingaki S. Clinical usefulness of real-time tissue elastography in the evaluation of cervical lymph node metastases in patients with oral cancer. Head and Neck Cancer 2008;34:518.

Tan R, Xiao Y, He Q. Ultrasound elastography: Its potential role in assessment of cervical lymphadenopathy. Acad Radiol 2010;17:849-55.

Thomas A, Degenhardt F, Farrokh A, Wojcinski S, Slowinski T, Fischer T. Significant differentiation of focal breast lesions: calculation of strain ratio in breast sonoelastography. Acad Radiol 2010;17:558-63.

Thomas A, Kummel S, Gemeinhardt O, Fischer T. Real-time sonoelastography of the cervix: tissue elasticity of the normal and abnormal cervix. Acad Radiol 2007;14:193-200.

Zhi H, Xiao XY, Yang HY, Ou B, Wen YL, Luo BM. Ultrasonic elastography in breast cancer diagnosis: strain ratio vs 5-point scale. Acad Radiol 2010;17:1227-33.

Zhu QL, Jiang YX, Liu JB, Liu H, Sun Q, Dai Q, Chen X. Real-time ultrasound elastography: its potential role in assessment of breast lesions. Ultrasound Med Biol 2008;34:1232-8. 
Fig.1

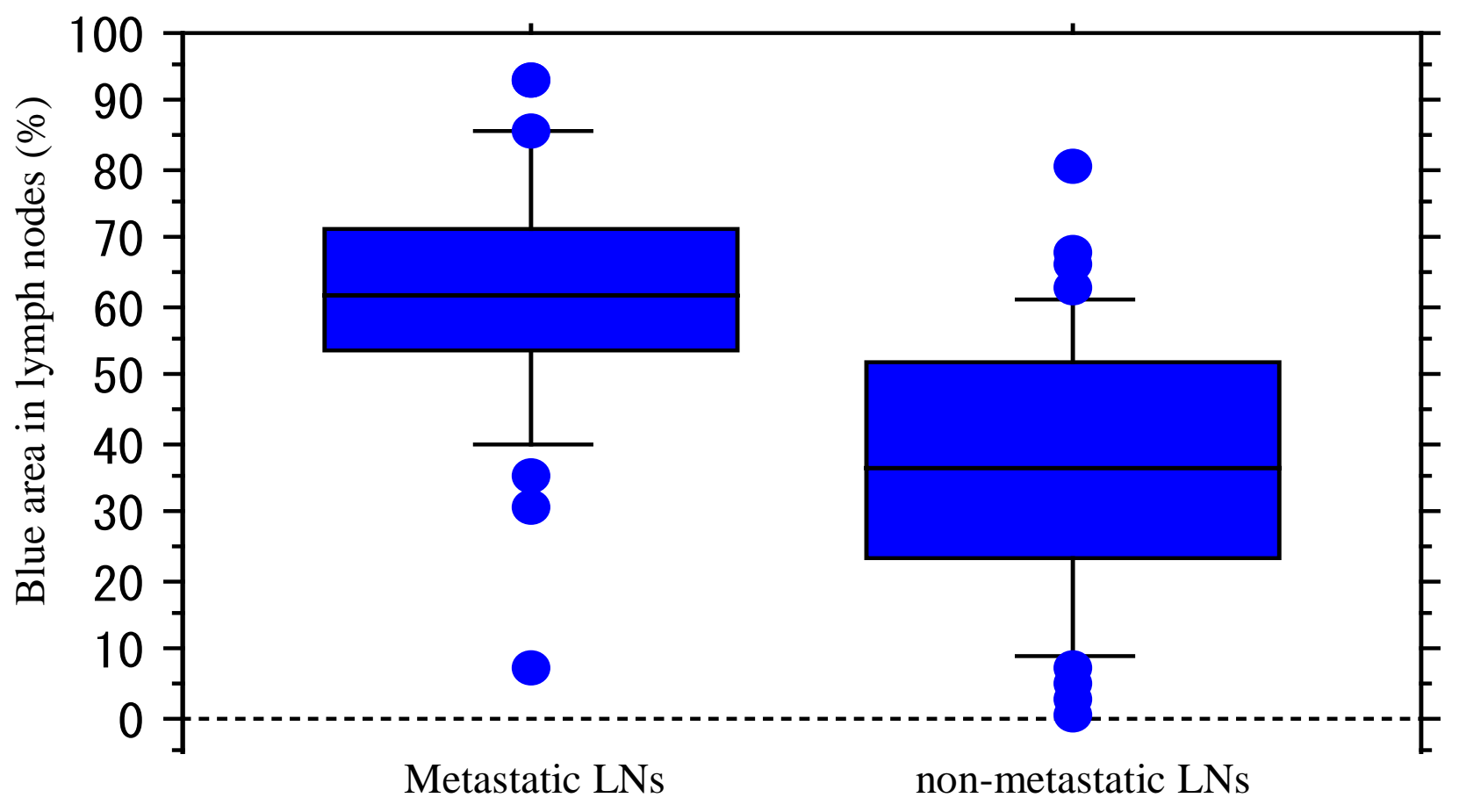


Table1. Elastography scoring system

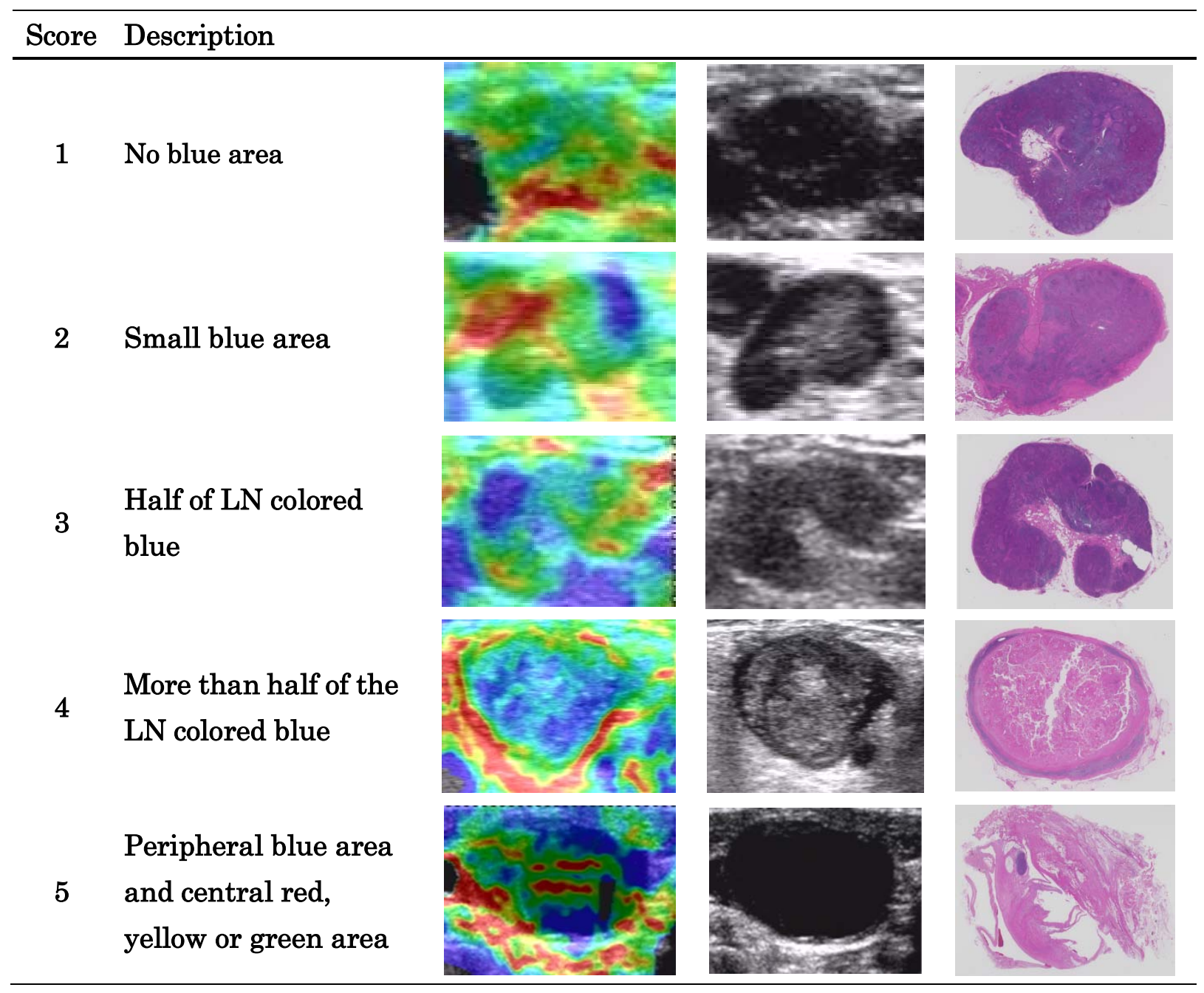


Table 2. Relationship between histological examination and US B-mode or color Doppler parameters.

Histological examination

US parameters

Metastatic $(n=31) \quad$ Non-metastatic $(n=40)$ P value

B-mode

Minimal diameter (mn

$9.1(3.2-19.2)$

$4.0(2.4-8.3)$

$<0.01$

Minimal diameter

$8 \mathrm{~mm} \leqq$
$8 \mathrm{~mm}>$

16

1

$<0.01$

Shape index (Min/Mas

15

39

Margin

Smooth
Irregular

27

40

$<0.05$

Irregular

4

0

Pattern of internal echo

Homogeneous

14

40

$<0.01$

Heterogeneous

17

0

Echogenic hilus

Present

6

21

$<0.01$

Absent

25

19

Color Doppler

Blood flow

Present

12

17

n.s.*

Absent

15

7 
Table 3. Frequency and percentage of LN elastography scores according to histological examination.

\begin{tabular}{cccc} 
& \multicolumn{2}{c}{ Histological examination } & \\
\cline { 2 - 3 } Elastography score & $\mathrm{n}=31$ & Non-metastatic & \\
\hline 1 & $1(3.2 \%)$ & $\mathrm{n}=40$ & $<0.01$ \\
2 & $4(12.9 \%)$ & $8(20 \%)$ & \\
3 & $5(16.1 \%)$ & $25(62.5 \%)$ & \\
4 & $17(54.9 \%)$ & $5(12.5 \%)$ & \\
5 & $4(12.9 \%)$ & $2(5 \%)$ & \\
\hline
\end{tabular}


Table 4. Comparison of elastographic-negative (elastography score of 1 or 2 ) and positive (score of 3 to 5) LNs with histological findings

Histological examination Elastographic negative Elastographic positive $\quad \mathrm{P}$ value

$\begin{array}{llll}\text { Metastatic }(\mathrm{n}=31) & 5 & 26 & <0.01\end{array}$

$\begin{array}{lll}\text { Non-metastatic }(\mathrm{n}=40) & 33 & 7\end{array}$

Elastographic negative group meaning elastography score 1,2.

Elastographic positive group meaning elastography score 3 to 5 . 
Table 5. Comparison of the percentage of blue area in LN elastography with histological examination.

\begin{tabular}{lccl} 
& \multicolumn{2}{c}{ Percentage of blue area in LN (\%) } & \\
\cline { 2 - 3 } Histological examination & $50>$ & $50 \leqq$ & P value \\
\hline Metastatic $(\mathrm{n}=31)$ & 7 & 24 & $<0.01$ \\
Non-metastatic $(\mathrm{n}=40)$ & 28 & 12 & \\
\hline
\end{tabular}


Table 6.Diagnostic performance of B-mode or elastographic evaluation, separately or in combination.

\begin{tabular}{lccc}
\hline & B-mode & Elastography & Combined \\
\hline Sensitivity (\%) & 70.97 & 83.87 & 90.32 \\
Specificity (\%) & 97.5 & 82.5 & 80 \\
Accuracy (\%) & 85.92 & 83.1 & 84.51 \\
Positive predictive value (\%) & 95.65 & 78.79 & 77.78 \\
Negative predictive value (\%) & 81.25 & 86.8 & 91.43 \\
\hline
\end{tabular}


Table 7. Logistic regression analysis of related factors

\begin{tabular}{lcc}
\hline Related factor & Hazard ratio $\left(95 \% \mathrm{CI}^{*}\right)$ & P value \\
\hline Minimal diameter & $2.19 \times 10\left(1.02-4.74 \times 10^{2}\right)$ & 0.05 \\
Shape index & $1.45\left(2.68 \times 10^{-1}-7.89\right)$ & 0.67 \\
Margin & $3.10 \times 10^{-2}$ & 1 \\
Pattern of internal echo & $2.95 \times 10^{6}$ & 1 \\
Echogenic hilus & $3.93 \times 10^{-1}\left(7.80 \times 10^{-2}-1.99\right)$ & 0.26 \\
Elastography score & $1.82 \times 10\left(2.79-1.19 \times 10^{2}\right)$ & $<0.01$ \\
Blue area in lymph node & $6.11 \times 10^{-1}\left(9.90 \times 10^{-2}-3.77\right)$ & 0.6 \\
\hline
\end{tabular}

* CI = confidence interval 